\title{
Dependence of impurity incorporation upon substrate misorientation during GaAs growth by metalorganic vapour phase epitaxy
}

\author{
P.R. Hageman *, J. te Nijenhuis, M.J. Anders, L.J. Giling \\ Department of Experimental Solid State Physics III, R.I.M., Faculty of Science, University of Nijmegen, Toernooiveld, 6525 ED Nijmegen, \\ The Netherlands
}

\begin{abstract}
Doping studies of the incorporation behaviour of three different dopants ( $\mathrm{Zn}, \mathrm{In}$ and $\mathrm{Si}$ ) versus the misorientation of the (100) surface during MOVPE growth of GaAs have been carried out with diethylzinc, trimethylindium and disilane as precursors. The incorporation of the dopants has been studied as function of the input mole fraction dopant, growth temperature, degree and direction of misorientation. In order to explain the results we discuss the BCF theory and the nature of the steps as function of above mentioned parameters. It appears that the BCF theory alone cannot explain the results, a counteracting mechanism has been introduced based on preferential arsenic desorption from the step edges.
\end{abstract}

\section{Introduction}

Since the first publications by Manasevit [1], MOVPE has developed into a mature crystal growth technique and is nowadays used for large scale production of devices. Because it results in the smoothest surfaces, MOVPE of GaAs is typically performed on slightly misoriented (100) surfaces. However, the effect of the direction and the magnitude of the misorientation on the different incorporation behaviour of dopant elements has scarcely been investigated, although differences up to $40 \%$ have been found in doping level for only a difference of a few degrees in misorientation [2,3]. Furthermore, the two different step configurations on the (100) surface give rise to anisotropic incorporation of doping species $[4,5]$.

\footnotetext{
* Corresponding author. Fax: +31 24 3652314; E-mail paulh@sci.kun.nl
}

We present a study on the orientation dependence of the incorporation process of three different dopant species in GaAs during MOVPE as a function of the growth temperature, the input mole fraction of the precursor and the amount and the direction of the misorientations of the GaAs (100) substrates. We used respectively an iso-electronic dopant (indium), a p-type dopant (zinc) and an amphoteric n-type dopant (silicon) each with their own incorporation mechanism [5-9].

\section{Experimental details}

GaAs epitaxial layers have been doped with In, $\mathrm{Zn}$ or $\mathrm{Si}$, by MOVPE (20 mbar) in a horizontal reactor at growth temperatures between 640 and $760^{\circ} \mathrm{C}$ on GaAs substrates. These semi-insulating substrates were all exactly (100) or misoriented by $2^{\circ}, 4^{\circ}$ or $6^{\circ}$ towards $(011)$ or towards $(01 \overline{1})$, i.e. 
towards the two major step directions. Trimethylgallium, trimethylindium, diethylzinc, disilane and arsine have been used as precursors. A constant TMGa partial pressure of $2.5 \times 10^{-3}$ mbar $(\mathrm{V} / \mathrm{III}$ ratio between 35 and 225 ) resulted in a growth rate between 0.2 and $0.3 \mu \mathrm{m} / \mathrm{min}$, depending on the growth temperature. The indium concentrations in the completely unrelaxed epitaxial layers were determined from photoluminescence spectra [10]. The carrier concentrations of the zinc- and silicon-doped samples were determined by Hall-Van der Pauw measurements.

\section{Results and discussion}

The epitaxial layers showed mirror-like surfaces at all growth conditions, even the strained In $\mathrm{Ga}_{1-\gamma}$ As layers, because their thicknesses were kept below the critical layer thickness for the formation of misfit dislocations [11]. The background concentration of undoped GaAs was low $\left(n \approx 5 \times 10^{14}\right.$ $\left.\mathrm{cm}^{-3}\right)$. No saturation was observed for the $\mathrm{Zn}$ - and Si-doping experiments $[6,7]$, justifying the assumption that the measured charge carrier concentration can be taken equal to the incorporated dopant concentration.

From studies on the temperature dependence of the dopant incorporation, we obtained apparent activation energies $\left(E_{\text {app }}\right)$ for the three different dopants ( $\mathrm{Zn}, \mathrm{Si}$ and $\mathrm{In}$ ), averaged over the used misorientations; no significant variations between the different orientations were observed.

An $E_{\text {app }}$ of $-59 \mathrm{kcal} \mathrm{mol}^{-1}$ is obtained for doping with DEZn. An adsorption/desorption equilibrium of zinc species at a step followed by step trapping is responsible for this incorporation behaviour [5,7]. This is supported by the fact that in a $\log -\log$ plot of hole concentration versus input mole fraction DEZn a linear behaviour is observed with a slope of 1 for all the used orientations [5].

Experiments with TMIn as dopant showed an $E_{\text {app }}$ of $9.7 \mathrm{kcal} \mathrm{mol}^{-1}$, which is explained by the presence of an adduct of TMIn and $\mathrm{AsH}_{3}$ at lower growth temperatures [12]. The small TMIn concentration is rate limiting for this adduct formation. This results in a reduced indium incorporation rate since the flux of the adduct to the surface is smaller because of its smaller diffusion coefficient and the larger Soret effect as compared to the monomethylindium species. At higher temperatures the adduct becomes less stable and decomposes with an enthalpy of $10 \mathrm{kcal} \mathrm{mol}^{-1}$, raising in this way the surface concentration of indium. The indium distribution coefficient increases from 0.65 (which is comparable to the value found by Kuo et al. [13]) to I when the growth temperature is increased from 640 to $760^{\circ} \mathrm{C}$. This indicates that only at the highest temperature step trapping is possible.

For disilane as silicon dopant, not a surface equilibrium, but homogeneous gas phase kinetics are rate determining for the incorporation [6]. The obtained $E_{\text {app }}$ of $32 \mathrm{kcal} \mathrm{mol}^{-1}$ is determined by the gas phase decomposition of disilane into $\mathrm{SiH}_{2}$ coupled to diffusion of this species towards the growing surface at 20 mbar reactor pressure [6]. Theoretically one can calculate a value of about $38 \mathrm{kcal} \mathrm{mol}^{-1}$ for this case, which is within the uncertainty limits of the experiments and the literature values for reaction rates and diffusion constants [6].

Before discussing the incorporation behaviour versus misorientation, a short description of the three factors responsible for the anisotropic behaviour of the dopant incorporation will be given.

(i) Kink density. The steps in the two major directions on the (100) surface behave differently under arsenic-rich conditions during MOVPE due to surface reconstruction $[14,15]$. The [011] step is fully kinked, i.e. its step front is rough, whereas the [011] step is a stable step with a straight step front and only an occasional kink site. Each dopant species arriving at a group III site at the [011] step can form a triple bond at any step site and is directly incorpo-

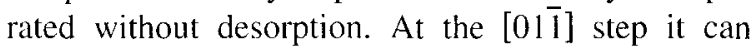
only adsorb by the formation of a single bond, and will therefore diffuse along the step until it arrives at a specific kink site [16] where a double bond can be formed. Diffusion along the step or back to the surface, where desorption is possible, is favoured above direct incorporation at this step [17].

(ii) The arsenic coverage of the surface. The arsenic coverage is decreased whenever (a) the growth temperature is increased, (b) the $\mathrm{V} / \mathrm{III}$ ratio is decreased thereby reducing the surface reconstruction by the arsenic dimers or (c) by an increase of the step density. Desorption from the [011] step is 
relatively easy and leads to a smaller number of kink sites. The $[01 \overline{1}]$ step coverage is hardly influenced by the arsenic partial pressure. At higher misorientations the step becomes rougher due to the preferential arsenic evaporation from the steps [18], even at lower temperatures, thereby decreasing the number of available kink sites for group III species considerably.

(iii) Diffusion fluxes towards the steps. It was shown that, according to the BCF theory [19], in the case that the dopant incorporation is determined by an adsorption/desorption equilibrium, there will be an increase with increasing misorientation angle of the incorporation of that species with the lowest binding energy with arsenic [5]. This will hold for both the incorporation of zinc and of indium because the binding energy with arsenic of indium $(35.2 \mathrm{kcal}$ $\mathrm{mol}^{-1}$ [20]) and that of zinc (about $30 \mathrm{kcal} \mathrm{mol}^{-1}$ [7]) are smaller than with gallium $\left(39.4 \mathrm{kcal} \mathrm{mol}^{-1}\right.$ [5]), in contrast to that of silicon (55 kcal mol ${ }^{-1}$ [4]). In this case a decrease in incorporation should be observed.

We will now discuss the results of the incorporation behaviour versus the misorientation for the different dopants. In Fig. 1 the hole concentration, as obtained after zinc doping, is given versus the misorientation angle for misorientations towards (011) and $(01 \overline{1})$ in a semi-logarithmic plot. The exactly oriented samples always exhibit the highest zinc concentration and the dependence on misorientation

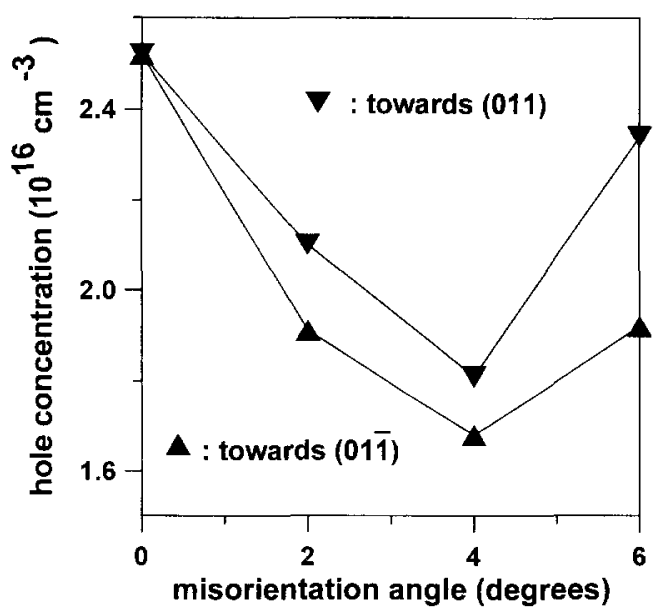

Fig. 1. Hole concentration of zinc-doped GaAs versus misorientation angle towards $(011)$ and towards $(0 \overline{11})$ for $T_{\text {growth }}=720^{\circ} \mathrm{C}$ and an input mole fraction DEZn of $3.5 \times 10^{-6}$. is the same for both directions, with a minimum at $4^{\circ}$ off. The offset between the two directions of misorientation is determined by the step configurations as will be discussed below in the case of indium incorporation. The BCF theory cannot explain this minimum in the incorporation of zinc. The incorporation of zinc is determined by the adsorption/desorption equilibrium which causes a constant concentration of zinc species at the surface at constant conditions. At constant vertical growth rates, higher misorientations lead to lower step velocities thereby diminishing step trapping of zinc species, i.e. less zinc is incorporated. However, a larger misorientation also leads to higher step densities, thereby increasing the number of kink sites. Consequently, this results in a higher concentration of zinc at kink positions, so the incorporation will be enhanced. The competition between these two mechanisms (BCF diffusion and preferential arsenic desorption) leads to the observed minimum. From Fig. 1 it is also observed that the arsenic desorption at higher misorientations towards $(01 \overline{1})$ (see below) does not introduce much difference in zinc incorporation. Apparently the zinc incorporation shows no strong dependence on the arsenic coverage at the step edges in contrast to the indium incorporation.

The indium incorporation during MOVPE of GaAs versus the misorientation towards (011) and $(01 \overline{1})$ is shown in Fig. 2 for four different growth temperatures. At $640^{\circ} \mathrm{C}$ the indium concentration in the $(100) 2^{\circ}(011)$ oriented sample is higher than that in the $(100) 2^{\circ}(01 \overline{1})$ orientation, whereas for higher growth temperatures these differences decrease and finally at $760^{\circ} \mathrm{C}$ the situation is reversed. This can be explained by considering the nature of the steps carefully. At $640^{\circ} \mathrm{C}$, i.e. a high arsenic surface coverage, the $\mathrm{Ga}$ and In growth species are directly incorporated at step sites at the [011] step; no desorption from it is possible, only from the terraces. In contrast, desorption from the $[01 \overline{1}]$ steps is possible, whereby In preferentially desorbs over $\mathrm{Ga}$ because of bond strengths [20] resulting in a lower In incorporation on the $2^{\circ}[01 \overline{1}]$ as compared to the $2^{\circ}[011]$. Raising the growth temperature reduces the number of kink sites on the [011] step leading to lower In incorporation as compared to the [01-1] step.

The indium incorporation versus misorientation shows the same trend as the zinc incorporation (see 


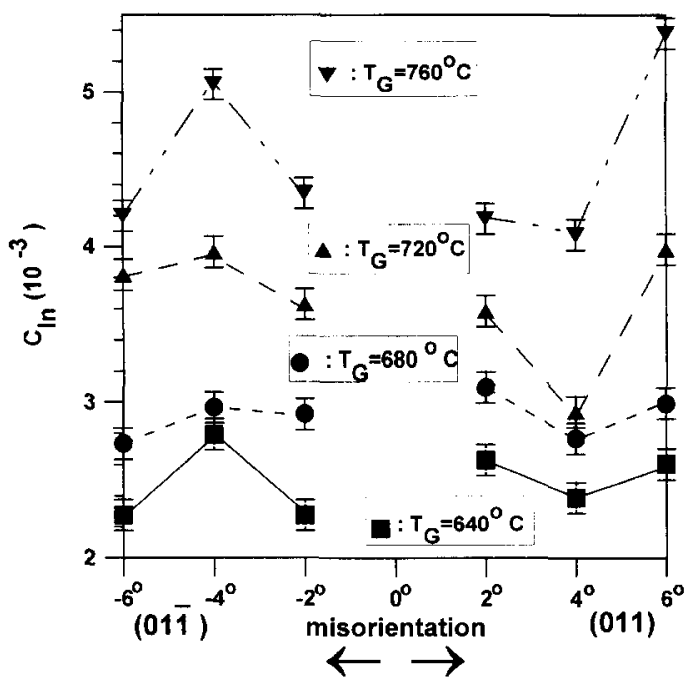

Fig. 2. Indium concentration in the $\operatorname{In}_{x} \mathrm{Ga}_{1-x}$ As epitaxial layers, $x_{4}$, grown at four different temperatures, as a function of the misorientations towards $(01 \overline{1})$ and (011). An input mole fraction TMIn of $4.34 \times 10^{-7}$, as compared to an input mole fraction of TMGa of $1.25 \times 10^{-4}$, was used. The reproducibility of the data is indicated by the error bar.

Fig. 1); for misorientations towards (011) a minimum is observed for $4^{\circ}$ off; for the (01) direction it is reversed, i.e. a maximum is observed for this sample. This again points to two counteracting mechanisms determining the incorporation so that the minimum respectively maximum can be explained.

For all four growth temperatures the trends in Fig. 2 are identical. Given the fact that only at the highest temperature step trapping might be possible, these observations exclude this mechanism. The decrease in the indium concentration observed in the layers $4^{\circ}$ misoriented towards (011) illustrates that the indium incorporation is not determined by the adsorption/desorption equilibrium. The group III growth species are directly incorporated upon arrival at this step, meaning that the BCF-flux theory is not applicable at these low misorientations. Furthermore, as was observed from the temperature series of the $2^{\circ}$ samples the arsenic desorption from this step is much easier compared to the step in the [011] direction. Consequently, here the effect of preferential desorption of arsenic from the step edges is already observed at low misorientation angles. Only at high misorientations can the competition between the fluxes of indium and gallium as derived from the BCF theory become clear, as so much arsenic has desorbed that also here the adsorption/desorption equilibrium of indium at the step can establish itself, raising in this way the indium concentration for all temperatures.

For misorientations larger than $2^{\circ}$ the competition between the gallium and indium fluxes - as discussed above using the BCF theory - leads to an increase in indium. The increase of indium between $2^{\circ}$ and $4^{\circ}$ off towards $(01 \overline{1})$ indeed indicates that in this situation the indium incorporation proceeds via an adsorption /desorption equilibrium following from the BCF flux theory.

However, this flux model does not predict the decrease in indium incorporation observed at higher misorientations towards ( $01 \overline{1})$. Probably, this can be explained by the roughening of the [01] step, due to desorption of As as expected for higher misorientations, leading to a stronger preferential arsenic evaporation from the step edges and a decrease in the number of kink sites for In-species [18]. The higher step densities at higher misorientation angles require a higher arsenic partial pressure in order to maintain the full arsenic coverage of the steps as was assumed in the theory. Therefore, under the constant arsenic partial pressures the indium species arriving at the steps will find less kink sites, resulting in a decrease in indium concentration in the layer. Apparently, this is not counteracted by the increase in kink concentration due to the higher step density.

The effect of step bunching is reflected in Fig. 2 by the small differences between incorporated indium at the low growth temperatures at the various misorientations, whereas at the higher growth temperatures the effect of the misorientations becomes more pronounced [21].

In contrast to zinc- or indium-doped GaAs, less pronounced differences have been found for doping with silicon. In Fig. 3 the net carrier concentration as obtained from Hall measurements is given versus misorientation. For different $\mathrm{V} / \mathrm{III}$ ratios at $760^{\circ} \mathrm{C}$ the carrier concentration decreases in $\mathrm{V} / \mathrm{III}$ order $n_{35 \text { or } 75}>n_{125}>n_{225}$. Step trapping, BCF theory or arsenic desorption cannot account for these results because then a decrease in carrier concentration is expected.

This disagrees with the results of Tang et al. [4], 


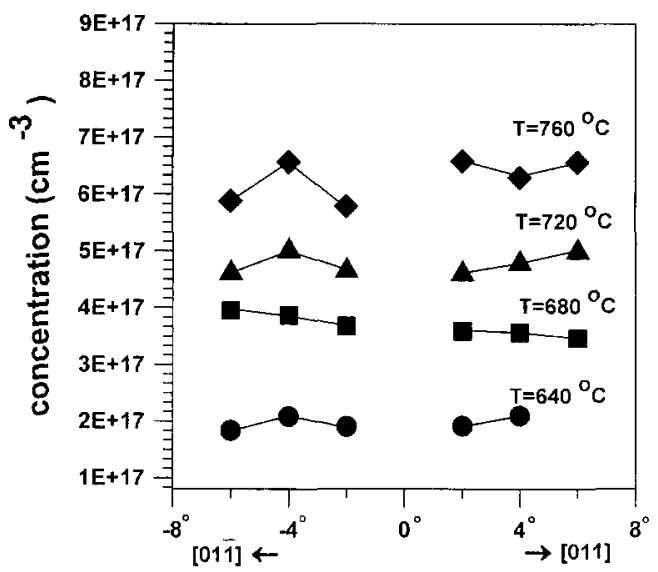

Fig. 3. Carrier concentration in the silicon-doped GaAs epitaxial layers for the growth at four different temperatures, as a function of the misorientations towards (01T) and (011). An input mole fraction $\mathrm{Si}_{2} \mathrm{H}_{6}$ of $2 \times 10^{-i)}$ was used with a $\mathrm{V} /$ III ratio of 125 .

who reported that at atmospheric pressure with increasing misorientation angle less silicon is incorporated because of a diminishing of the effect of step trapping and that an increasing $\mathrm{V} / \mathrm{III}$ ratio leads to an increase in silicon incorporation. Our experiments are supported by the results of Thompson [22] who found at growth temperatures above $650^{\circ} \mathrm{C}$ hardly any influence of the misorientation. This is explained by the formation of $\mathrm{SiH}_{3} \mathrm{AsH}_{2}$ in the gas phase and the chemisorption of it on the surface followed by diffusion over the surface where it can desorb or encounter a step. Trapped at a step it can release an $\mathrm{AsH}_{3}$ and incorporate a silicon atom. Alternatively, the reverse is also possible: incorporation of As and desorption of $\mathrm{SiH}_{2}$. This mechanism is supposed to be temperature dependent whereby at lower temperatures the release of $\mathrm{SiH}_{2}$ is more likely and at higher temperatures the incorporation of $\mathrm{Si}$ is dominating. In both cases, doping with indium and silicon, the adduct formation levels off the influence of the misorientation on the incorporation rate due to the Soret effect. At high $\mathrm{V} /$ III ratios the competition between $\mathrm{AsH}_{3}$ and $\mathrm{SiH}_{3} \mathrm{AsH}_{2}$ molecules for adsorption on the surface shifts towards adsorption of arsine, thereby decreasing the silicon incorporation. It is suggested that at higher reactor pressures this reaction between arsine and (di-)silane is of less importance because the total amount of arsine in the reactor is considerably reduced, thereby diminishing the influence of the adduct formation explaining the results of Tang et al. [4].

\section{Conclusions}

Dependence upon the misorientation of the zinc, indium and silicon incorporation during MOVPE growth of GaAs on vicinal (100) substrates has been studied. Gas phase chemistry and adsorption/desorption equilibria have been used to explain the temperature dependence of the incorporation. The incorporation as function of the misorientation has been explained by the introduction of competitive mechanisms based on preferential desorption of arsenic from the step edges, which counteract the $\mathrm{BCF}$ theory.

\section{Acknowledgements}

S.M. Olsthoorn (photoluminescence experiments), G.J. Bauhuis (electrical characterization) and W.J.P. van Enckevort (discussions) are acknowledged. This work has been performed as a part of the research programme of the Nederlandse Maatschappij voor Energie en Milieu (NOVEM).

\section{References}

[1] H.M. Manasevit, J. Electrochem. Soc. 116 (1968) 1725

[2] X. Tang, H.G.M. Lochs. P.R. Hageman. M.H.J.M. de Croon. L.J. Giling and A.J. Bons, J. Crystal Growth 98 (1988) 827.

[3] T. Nakanisi, J. Crystal Growth 68 (1984) 282.

[4] X. Tang, J. te Nijenhuis, Y. Li and L.J. Giling, J. Crystal Growth 107 (1991) 263.

[5] M.J. Anders, P.R. Hageman and L.J. Giling, J. Crystal Growth 142 (1994) 292.

[6] P.R. Hageman. M.H.J.M. de Croon, J.N.H. Reek and L.J. Giling, J. Crystal Growth 116 (1992) 169.

[7] P.R. Hageman, M.H.J.M. de Croon, X. Tang and L.J. Giling, J. Crystal Growth 129 (1993) 281.

[8] T. Dosluoglu, U. Sudarsan and R. Solanki, J. Crystal Growth $106(1990) 643$.

[9] J. te Nijenhuis, P.R. Hageman and L.J. Giling. J. Crystal Growth 167 (1996) 397.

[10] J. te Nijenhuis, P.J. van der Wel, R.W.F. van Asten, P.R. Hageman and L.J. Giling, J. Crystal Growth 107 (1991) 496. 
[11] J.W. Matthews and A.E. Blakeslee, J. Crystal Growth 27 (1974) 118.

[12] M.J. Ludowise, J. Appl. Phys. 58 (1985) R31, and references therein.

[13] C.P. Kuo, R.M. Cohen, K.L. Fry and G.B. Stringfellow, J. Electron. Mater. 14 (1985) 231.

[14] H. Asai, J. Crystal Growth 80 (1987) 425.

[15] L.J. Giling and W.J.P. van Enckevort, Surf. Sci. 161 (1985) 567.

[16] T. Fukui and H. Saito, J. Vacuum Sci. Technol. B 6 (1988) 1373.
[17] L.J. Giling and M.H.J.M. de Croon, J. Crystal Growth 107 (1991) 56.

[18] J.-S. Lee, S. Iwai, H. Isshiki, T. Meguro, T. Sugano and Y. Aoyagi, Appl. Phys. Lett. 67 (1995) 1283.

[19] W.K. Burton, N. Cabrera and F.C. Frank, Philos. Trans. R. Soc. London A 243 (1951) 299.

[20] T. Nishinaga and K.-I. Cho, Jpn. J. Appl. Phys. 27 (1988) L12.

[21] M. Kasu and N. Kobayashi, J. Appl. Phys. 78 (1995) 3026.

[22] A.G. Thompson, J. Crystal Growth 124 (1992) 457. 